Results The level of MPO in CHD group was significantly higher than that in the control group, and the level of PON level was significantly lower than that in the control group. In ACS group, MPO level was also significantly higher than that in SAP group, and the PON level was significantly lower than that in the SAP group. When the CHD is more severer, the MPO level will be more higher and the level of PON will be more lower. With coronary artery disease type and the severity of coronary artery disease, MPO levels increased gradually, while the PON level decreased gradually, stepwise regression analysis showed that $\mathrm{MPO}, \mathrm{PON}, \mathrm{HDL}, \mathrm{LDL}$ concentrations were related significantly with the Gensini score of coronary lesions.

Conclusion MPO, PON1 would be better indicators to reflect the local inflammation of atherosclerotic plaque, LDL can affect the conversion of ox-LDL by MPO, PON1. So that, it can be the key mechanism of atherosclerotic plaque's stability. Through detection of serum pro-inflammatory factor (MPO), and anti-inflammatory factor (PON1) levels in CHD patients and investigate their relationship with CHD and coronary artery disease, further analysis of the imbalance of pro-inflammatory cytokines and anti-inflammatory factor may be particularly important in the pathological significance in coronary heart disease, especially in acute coronary syndrome, and provide a new basis for the diagnosis and prediction of coronary heart disease and coronary artery disease.

\section{e0380 ASSOCIATIONS BETWEEN PLASMA N-TERMINAL PRO BRAIN NATRIURETIC PEPTIDE AND OXIDATIVE STRESS IN PATIENTS WITH CHRONIC STABLE CORONARY ARTERY DISEASE}

doi:10.1136/hrt.2010.208967.380

Liu Fucheng, Chen Luyuan. Guangdong Cardiovacular Institute, Guangdong, China

Objectives The present study was aimed to explore whether plasma $\mathrm{N}$-terminal pro brain natriuretic peptide was associated with oxidative stress in patients with chronic stable coronary artery disease.

Methods Plasma levels of $\mathrm{N}$-terminal pro brain natriuretic peptide, hemeoxygenase-1 and oxidative low-density lipoprotein were determined by electrochemiluminescence and ELISA respectively in ninety-four patients with chronic stable coronary artery disease and 35 controls without coronary artery disease confirmed by coronary angiography. Correlations were analysed among these three biochemical marks.

Results Logarithms-transformed levels of $\mathrm{N}$-terminal pro brain natriuretic peptide and oxidative low-density lipoprotein in patients with chronic stable coronary artery disease were both higher than the controls $(5.01 \pm 0.14$ vs $3.73 \pm 0.16 \mathrm{Ln} \mathrm{mg} / 1, \mathrm{p}<0.001 ; 2.38 \pm 0.09$ vs $0.09 \pm 0.13$ Ln ug/l, $\mathrm{p}<0.001)$, whereas squart-root-transformed level of hemeoxygenase- 1 was lower than the controls $(6.10 \pm 0.24$ vs $13.96 \pm 0.86$ Sqrt ug/l, $p<0.001)$. The difference in these three biochemical marks between the two groups was still in presence after being adjusted by age, sex, blood glucose, lipids and left ventricular ejection fraction. However, there wasn't difference in them in subgroup analysis by coronary artery lesion count. Logarithms-transformed levels of $\mathrm{N}$-terminal pro brain natriuretic peptide and oxidative low-density lipoprotein were associated with each other positively $(r=0.281, p<0.05)$, whereas logarithms-transformed level of $\mathrm{N}$-terminal pro brain natriuretic peptide was correlated to squart-root-transformed level of hemeoxygenase-1 negatively $(r=-0.277, p<0.05)$. Squart-root-transformed level of hemeoxygenase- 1 was associated with logarithms-transformed level of oxidative low-density lipoprotein negatively ( $\mathrm{r}=-0.575$, $p<0.001)$. After being adjusted by age, sex, blood glucose, lipids and left ventricular ejection fraction, logarithms-transformed level of $\mathrm{N}$-terminal pro brain natriuretic peptide was still associated with logarithms-transformed level of oxidative low-density lipoprotein positively $(r=0.269, p<0.05)$ and with squart-root-transformed level of hemeoxygenase- 1 negatively $(r=-0.261, p<0.05)$.

Conclusions $\mathrm{N}$-terminal pro brain natriuretic peptide was closely associated with oxidative stress in patients with chronic stable coronary artery disease. However the details of the interaction between brain natriurectic peptide and oxidative stress remain unknown.

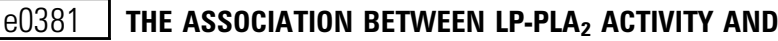 MAJOR CARDIOVASCULAR EVENTS IN ACUTE CORONARY SYNDROME AT THE CHINESE COMMUNITY}

doi:10.1136/hrt.2010.208967.381

Li Ning, Li Shuijun, Yu Chen, Gu Shuiming. Shanghai Xuhui Central Hospital

Objective Lipoprotein-associated phospholipase $A_{2}$ (Lp-PLA $A_{2}$ ) is a useful inflammatory marker of cardiovascular risk, but there are only few reports of its prognostic significance as a risk factor for acute coronary syndrome (ACS). It is necessary to evaluate the association of Lp-PLA $A_{2}$ with major cardiovascular events (MCVE) in patients with ACS and assess its incremental value for risk discrimination over established risk factors and biomarkers.

Methods 152 patients with ACS and one hundred forty-two patients without coronary artery disease (CAD) from Shanghai Xuhui District (aged <80 years) were enrolled from February 2007 to March 2008 and followed for a median of 6 months (4-10 months). Plasma Lp-PLA 2 activity was measured at baseline with liquid chromatography tandem mass spectrometry. Its clinical significance was evaluated with existing risk indicators.

Results Lp-PLA 2 activity was higher in patients with ACS than that in patients without CAD $(22.36 \pm 1.23 \mathrm{mg} / \mathrm{ml}$ vs $19.74 \pm 3.85 \mathrm{mg} / \mathrm{ml}$; $\mathrm{p}=0.027$ ). During the follow-up period, 5 cases of cardiovascular death, 8 cases of non-fatal myocardial infarction, and 11 cases of target vessel revascularization occurred. Elevated Lp-PLA $\mathrm{P}_{2}$ was associated with an increased risk of MCVE (HR, 1.52; 95\% CI, 1.09 to $2.37 ; p=0.033$ ). The Lp-PLA 2 activity level in incidental cases was higher than that in non-incidental cases $(p=0.04)$.

Conclusion In this community-based cohort of patients with ACS, Lp-PLA 2 was strongly and independently associated with major cardiovascular events and contributed incrementally to risk discrimination.

\section{e0382 RELATIONSHIP BETWEEN BRAIN NATRIURETIC PEPTIDE LEVELS IN PATIENTS WITH ACUTE CORONARY SYNDROME AFTER PERCUTANEOUS CORONARY INTERVENTION AND CARDIOVASCULAR EVENTS}

doi:10.1136/hrt.2010.208967.382

Liao Wei, Zhou Aiqin. The Affiliated Hospital of Gannan Medical College

Objective To analyse the relationship between in patients with acute coronary syndrome (ACS) at $48 \mathrm{~h}$ after percutaneous coronary intervention (PCI) and the relationship between the variation of brain natriuretic peptide (BNP) and the short and long-term cardiovascular events.

Methods 156 patients with ACS were given conventional preoperative and postoperative medication and standardised PC1. Plasma levels of BNP were measured at $48 \mathrm{~h}$ after operations. All patients were divided into two groups according to the level of BNP: A group 